Research Paper

\title{
Biodegradable Conduit Small Gap Tubulization for Peripheral Nerve Mutilation: A Substitute for Tradi- tional Epineurial Neurorrhaphy
}

\author{
Peixun Zhang ${ }^{1 凶}$, Na Han ${ }^{1}$, Tianbing Wang ${ }^{1}$, Feng Xue ${ }^{1}$, Yuhui Kou ${ }^{1}$, Yanhua Wang ${ }^{1}$, Xiaofeng Yin ${ }^{1}$, Laijin

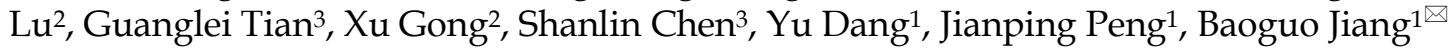

1. Department of Trauma \& Orthopedics, Peking University People's Hospital, Beijing, China

2. Department of Orthopedics, First Hospital of Jilin University, Changchun, China

3. Department of Orthopedics, Beijing Jishuitan Hospital, Beijing, China

\begin{abstract}
$\bowtie$ Corresponding author: Peixun Zhang, E-mail: zhangpeixun@bjmu.edu.cn; Baoguo Jiang, E-mail: jiangbaoguo@vip.sina.com
(c) Ivyspring International Publisher. This is an open-access article distributed under the terms of the Creative Commons License (http://creativecommons.org/ licenses/by-nc-nd/3.0/). Reproduction is permitted for personal, noncommercial use, provided that the article is in whole, unmodified, and properly cited.
\end{abstract}

Received: 2012.09.28; Accepted: 2012.12.24; Published: 2013.01.05

\begin{abstract}
Nerve regeneration and re-innervation are usually difficult after peripheral nerve injury. Epineurium neurorrhaphy to recover the nerve continuity is the traditional choice of peripheral nerve mutilation without nerve defects, whereas the functional recovery remains quite unsatisfactory. Based on previous research in SD rats and Rhesus Monkeys, a multiple centers clinical trial about biodegradable conduit small gap tubulization for peripheral nerve mutilation to substitute traditional epineurial neurorrhaphy was carried out. Herein, the authors reviewed the literature that focused on peripheral nerve injury and possible clinical application, and confirmed the clinical possibilities of biodegradable conduit small gap tubulization to substitute traditional epineurial neurorrhaphy for peripheral nerve mutilation. The biodegradable conduit small gap tubulization to substitute traditional epineurial neurorrhaphy for peripheral nerve mutilation may be a revolutionary innovation in peripheral nerve injury and repair field.
\end{abstract}

Key words: Biodegradable Conduit; Epineurial Neurorrhaphy; Peripheral Nerve Mutilation; Small Gap; Tubulization

\section{Introduction}

Peripheral nerve injury is a common casualty. Epineurial neurorrhaphy and lamellar sheath suture are clinically traditional repair methods after peripheral nerve injury. The clinical functional recovery is unsatisfactory, because neither epineurial neurorrhaphy nor lamellar sheath suture can resolve the problem of mis-connection of sensory and motor nerve fiber during the regeneration and re-innervation process. ${ }^{1,2}$

Based on the selective regeneration phenomenon of Y-shape tube peripheral nerve, many researchers had focused on the peripheral nerve selective regeneration phenomenon and its possible application.3,4
We had authenticated this selective regeneration phenomenon and tried different gap between the two ruptured stumps to find the most suitable gap in conduit, in order to reduce the selective regeneration effect maximatily in rats and rhesus Monkeys. Biological degradable conduit was used to repair peripheral nerve injury with $2 \mathrm{~mm}$ small gap between the two ruptured stumps and found that the repair effect was quite better than the traditional epineurial neurorrhaphy in a mimic clinical injury model.5-8 Here, we only used this method to repair nerve injury, but not nerve defect. After series of experiments in rats and rhesus monkeys, we started the clinical trial 
in human being to confirm this superiority of biodegradable conduit small gap tubulization to traditional epineurial neurorrhaphy and the possibility of substituting the traditional epineurial neurorrhaphy with biodegradable conduit small gap tubulization to repair nerve injury in clinical practice. The series of experiments confirmed the better regeneration effect of biodegradable conduit small gap $(2 \mathrm{~mm})$ tubulization for peripheral nerve injury than traditional epineurial neurorrhaphy, and this new methods which had not been reported previously will be brought into clinical practice for peripheral nerve injury.

\section{Materials and Methods}

\section{Patients}

A total of 50 patients with totally (median nerve, ulnar nerve, radius nerve) peripheral nerve injury at bilateral upper arms in three hospitals around China from Nov. 11, 2008 to Feb. 28, 2010 (Peking University People's Hospital; Beijing Jishuitan Hospital; Jilin University First Hospital) were enrolled in this study. All the enrolled patients aged from 22 to 46 years old were diagnosed as open injury with nerve totally fresh sharp un-destructive injury but without defect, and can be sutured directly. The clinical trial protocol was approved by the Institutional Review Boards of Chinese National Drug Administration and the Scientific Ethics Committees of each hospital. After obtaining the signed informed consent, patients were randomly divided into experimental group and control group using random number methods. This study was supervised by the Advisory Committee of Nerve Regeneration Conduit Clinical Trial and the study protocol conformed to the ethical guidelines of the 1975 Declaration of Helsinki. This clinical trial had been registered in Chinese Clinical Trial Registry

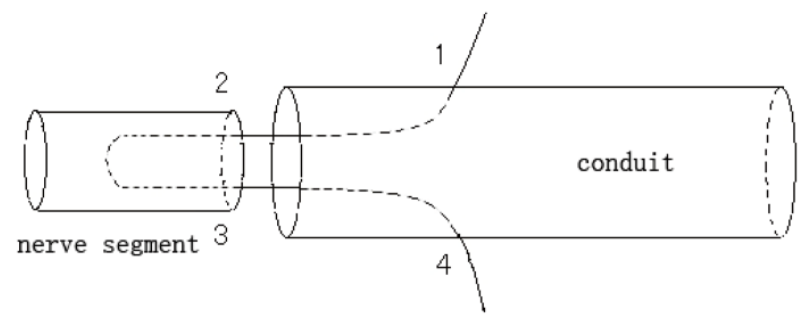

Center and the assigned number was ChiCTR-TNRC-10000801.

\section{Conduits}

Hollow cylindrical conduit (a de-acetyl chitin conduit invented by Beijing University People's Hospital and the Chinese Textile Academy, the State Patent No. 01136314.2). Size: tube length $10 \mathrm{~mm}$, thickness $1 \mathrm{~mm}$, inner diameter $4-6 \mathrm{~mm}$. Half quality degradation time of this biodegradable conduit material is six months.

\section{Methods}

According to the clinical trial profile, we used the random numbers to assign the 50 patients to different groups, where 25 patients received traditional epineurial neurorrhaphy and the other 25 patients received biodegradable conduit small gap tubulization to repair nerve injury. The operated surgeon in each hospital was the same surgeon, and the inspector of the Advisory Committee of Nerve Regeneration Conduit Clinical Trial told the surgeon to apply traditional epineurial neurorrhaphy or biodegradable conduit small gap tubulization by randomized number methods. We assessed the nerve regeneration functional recovery by clinical examination at 1 st month, 2nd month, 4th month, 6th month and electrophysiologic study at 6 month after first operation. The patients and clinical functional assessment staff were masked to the nature of treatment.

Traditional epineurial neurorrhaphy was carried out as it was often applied clinically, namely, to suture directly two ruptured stumps using absorbable suture. The biodegradable conduit small gap tubulization was carried out by bridging the two ruptured stumps using the biodegradable conduit, and $2 \mathrm{~mm}$ small gap existed between the two ruptured stumps (Figure 1 and 2).

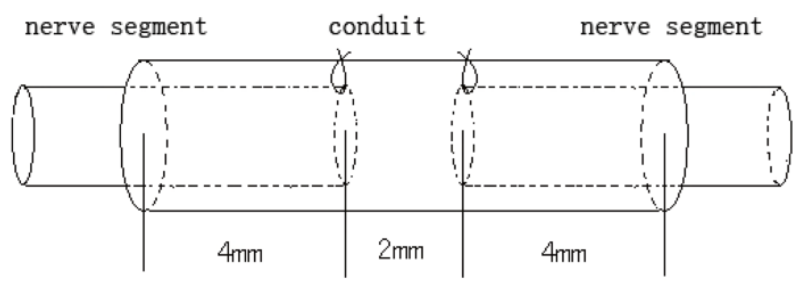

Figure I: The conduit small gap tubulization methods used in experimental group. The number I,2,3,4 was the suture needling sequence. When finished, there was $2 \mathrm{~mm}$ small gap remained between the two ruptured stumps. 


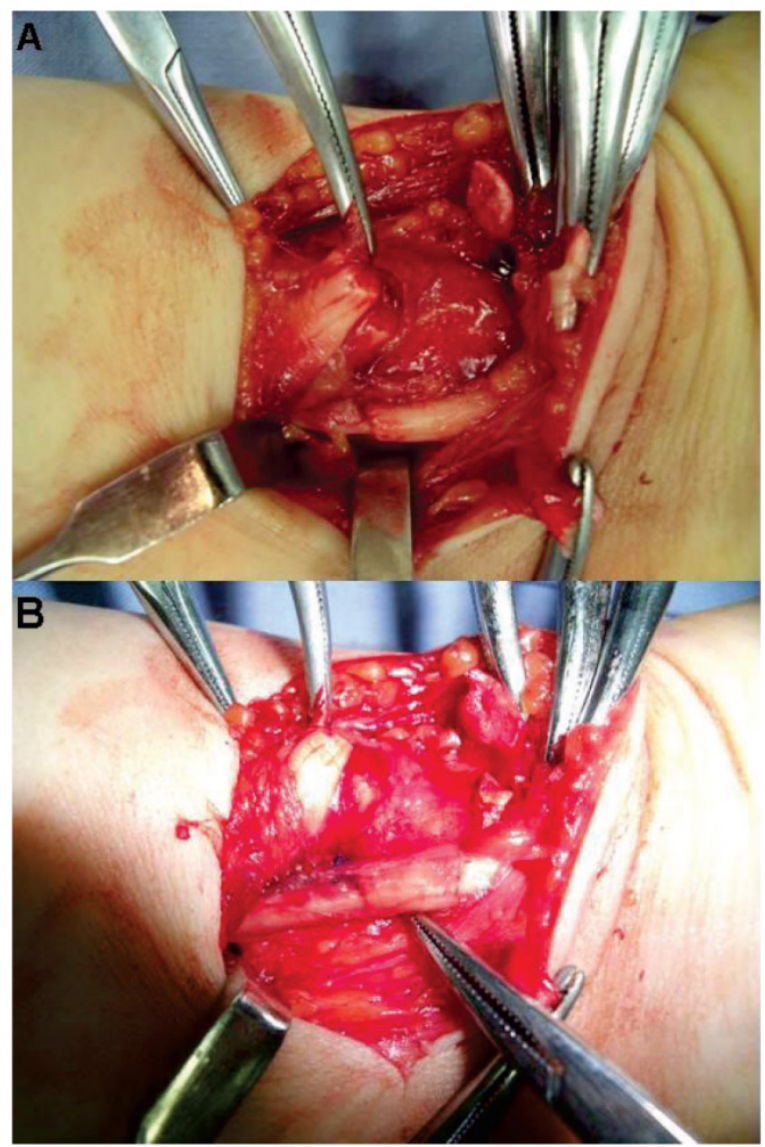

Figure 2: The biodegradable conduit small gap tubulization methods. This picture was taken from a patient who was diagnosed as right wrist totally ruptured median nerve and accepted the biodegradable conduit small gap tubulization methods to repair median nerve during operation. A. The ruptured median nerve stumps; $B$, the biodegradable conduit small gap tubulization.

\section{Statistical analysis}

The excellent and good criterion of two groups was compared using "t-test" statistical methods.

\section{Results}

\section{General Results}

No infection or anaphylactogenesis reaction complication was found in all the 50 enrolled patients during the four follow ups, which demonstrated the excellent biocompatibility. The operation procedure time of conduit small gap tubulization to repair peripheral nerve injury [(8.0 \pm 0.6$) \mathrm{min}]$ was shorter about $20 \%$ when compared with traditional epineurial neurorrhaphy [10.0 $\pm 0.5 \mathrm{~min}]$.

\section{Excellent and good criterion evaluation}

We adopted the combined evaluation of excellent and good criterion, established by Zhu Jiakai and Shen Ning Jiang, which involved the BMRC(British
Medical Research Council)motor function evaluation grading(M), sensory function grading(S) and autonomic nerve function evaluation grading(A). The combined evaluation of excellent and good rate of conduit small gap tubulization was much higher about $36.19 \%$ when compared with traditional epineurial neurorrhaphy at 6 month point, which implied significant difference.

As for the Visual analogue scale(VAS)assessment, there was significant difference between the experiment group and control group at 1st month (no neuropathic pain rate 91\%, $22.5 \%)$, 2nd month $(97 \%, 54.8 \%)$, 4th month $(100 \%$, $71.0 \%)$, 6th month $(100 \%, 77.4 \%)$ respectively, which implied that the conduit small gap tubulization to repair peripheral nerve injury can reduce neuropathic pain significantly compared with traditional epineurial neurorrhaphy.

\section{Electromyogram examinations}

Electromyogram examinations were carried out to evaluate the sense and motor nerve conduction recovery condition of injured peripheral nerve at 1st month, 2nd month, 4th month, 6th month after first operation. The sense and motor functional recovery rate of the experiment group and the control group at 6th month are shown in Table 2 and there was significant difference between the two groups.

Table I Comparison of combination evaluation excellent and good rate of two groups at four different follow up time

\begin{tabular}{llll}
\hline & \multicolumn{2}{c}{$\begin{array}{c}\text { The combination evaluation excellent } \\
\text { and good rate \% }\end{array}$} & P Value \\
\cline { 2 - 3 } & $\begin{array}{l}\text { Experiment } \\
\text { Group }\end{array}$ & Control Group & \\
\hline 1st month & 0 & 0 & - \\
2nd month & 0 & 0 & - \\
4th month & 21.43 & 2.5 & 0.015 \\
6th month & 76.19 & 40 & 0.001 \\
\hline
\end{tabular}

Table 2 Sensory and Motor Functional Recovery Rate

\begin{tabular}{|c|c|c|c|c|c|c|}
\hline \multirow[t]{3}{*}{ Time } & \multicolumn{2}{|c|}{$\begin{array}{l}\text { Rate of sensory con- } \\
\text { duction Recovery\% }\end{array}$} & \multirow[t]{3}{*}{ P1 } & \multicolumn{2}{|c|}{$\begin{array}{l}\text { Rate of motor conduc- } \\
\text { tion Recovery \% }\end{array}$} & \multirow[t]{3}{*}{ P2 } \\
\hline & Experimental & Control & & Experimental & Control & \\
\hline & Group & Group & & Group & Group & \\
\hline $\begin{array}{l}\text { 6th } \\
\text { month }\end{array}$ & 74.70 & 70.20 & 0.041 & 65.2 & 59.60 & 0.037 \\
\hline
\end{tabular}




\section{Discussion}

Peripheral nerve injury is very common clinically, although microsurgical technique has been noticeably improved during the last century, epineurial neurorrhaphy and interfascicular suture remain the main methods. If proximal nerve fibers grow into the wrong type of distal nerve fibers, taking motor nerve fiber connecting with sensory nerve for example, it will lead to failure of nerve regeneration and therefore affect the neurological function recovery. The peripheral nerve effective regeneration mainly depends on accurate docking of different nerve fibers and the re-innervation with target organ.

Based on the "Y-shape tube selective regeneration" phenomenon, ${ }^{3,4}$ we found that biological degradable conduit small gap tubulization can effectively promote peripheral nerve regeneration in rat and monkey, especially raptured stumps relative rotated. The degradable biological conduit small gap (2mm) tubulization for peripheral nerve injury provided a relative containing microenvironment and a new practical way in good docking of different nerve fibers during regeneration process. These research data inspired us to use this method to substitute traditional epineurial neurorrhaphy for repairing peripheral nerve injury. Although many researchers had reported the good functional recovery after applying conduit to repair the nerve defect, ${ }^{9-11}$ we designed this method to repair peripheral nerve injury, not for nerve defect. After getting the Chinese Government SFDA permission and Peking University People's Hospital Ethics Committee, we applied biological conduit small gap $(2 \mathrm{~mm})$ tubulization in human intending to confirm the possibility and feasibility of substituting traditional epineurial neurorrhaphy for repairing peripheral nerve injury. If the clinical results were positive the same as in rat and monkey, it may be a revolutionary improvement in peripheral nerve surgery field.

We adopted the sensory and motor functional recovery rate between the experimental value and control value (normal value or baseline value in different hospital center) to assess the functional recovery after operation. During the observation process, we found the operation procedure time of conduit small gap tubulization to repair peripheral nerve injury was shorter about $20 \%$ when compared with traditional epineurial neurorrhaphy. The operation process was very easy and concise. According to the combination evaluation excellent and good criterion, the combination evaluation excellent and good rate of conduit small gap tubulization was much higher about $36.19 \%$ when compared with traditional epi- neurial neurorrhaphy at 6th month point, which implied that the recovery effect of degradable biological conduit small gap tubulization was better than traditional epineurial neurorrhaphy.

Lundborg et $\mathrm{al}^{12}$ had used gel silica piece suture to construct conduit to repair median and ulnar nerves in the human forearm in 1997 and reported the early results. Compared with his report, our biodegradable conduit material and suture methods were different and neoteric. We chose $2 \mathrm{~mm}$ small gap based on "the Y-shape tube selective regeneration phenomenon" and our research results 13,14 while Lundborg et $\mathrm{al}^{12}$ chose 3-4 $\mathrm{mm}$ gap between the two injured stumps. Our functional assessment methods included BMRC standard (British Medical Research Council) motor function evaluation grading (M), sensory function grading $(S)$ and autonomic nerve function evaluation grading (A). As for the nerve conduction velocity recovery assessment, the nerve conduction recovery rate was chosen to assess the electromyogram recovery using the rate of experimental value to normal control value or baseline value in different clinical centers.

\section{Conclusion}

The operation procedure time of conduit small gap tubulization was more convenient and timesaving compared with traditional epineurial neurorrhaphy. The clinical regeneration effect of conduit small gap tubulization was better than traditional epineurial neurorrhaphy. Biological degradable conduit small gap tubulization was of possibility and feasibility to substitute traditional epineurial neurorrhaphy and become the most suitable method for peripheral nerve injury in clinical practice.

\section{Acknowledgement}

This research project was funded by Chinese National Natural Science Fund for Outstanding Youth (30625036), Chinese National Natural Science Fund(31171150, 31271284, 30801169, 81171146, 30971526), Beijing City Science \& Technology New Star ClassificationA-2008-10, Chinese Ministry of Education for Doctor Position New Teacher (20070001780), Chinese Educational Ministry New Century Excellent Talents Support Project (BMU20110270) and Peking University People's Hospital Project (RDB2009-01) and Chinese 973 Project Planning (2005CB522604).

\section{Competing Interests}

The authors have declared that no competing interest exists. 


\section{References}

1. Jiang B, Zhang P, Jiang B. Advances in small gap sleeve bridging peripheral nerve injury. Artif Cells Blood Substit Immobil Biotechnol 2010; 38:1-4.

2. Alluin $\mathrm{O}$, Wittmann $\mathrm{C}$, Marqueste $\mathrm{T}$, et al. Functional recovery after peripheral nerve injury and implantation of a collagen guide. Biomaterials 2009; 30:363-373.

3. Lundborg G. A 25-year perspective of peripheral nerve surgery: evolving neuroscientific concepts and clinical significance. J Hand Surg Am 2000; 25:391-414.

4. Siemionow M, Brzezicki G. Current techniques and concepts in peripheral nerve repair. Int Rev Neurobiol 2009;87:141-172

5. Zhang P, Zhang C, Kou Y, et al. The histological analysis of biological conduit sleeve bridging rhesus monkey median nerve injury with small gap. Artif Cells Blood Substit Immobil Biotechnol 2009;37:101-104

6. Zhang P, Yin X, Kou Y, et al. The electrophysiology analysis of biological Conduit sleeve bridgingRheus Monkey median nerve injury with small gap. Artif Cells Blood Substit Immobil Biotechnol 2008; 36:360-371.

7. Yu K, Zhang C, Wang Y, et al. The protective effects of small gap sleeve in bridging peripheral nerve mutilation. Artif Cells Blood Substit Immobil Biotechnol 2009; 37:257-264

8. Zhang $\mathrm{C}$, Zhang $\mathrm{P}$, Wang $\mathrm{Y}$, et al. Early spatiotemporal progress of myelinated nerve fiber regenerating through biological chitin conduit after injury. Artif Cells Blood Substit Immobil Biotechnol 2010; 38:103-108

9. Rustemeyer J, Dicke U. Prefabricated nerve conduits advance histomorphological and functional outcomes in nerve regeneration of the sciatic nerve of the rat. Int J Oral Maxillofac Surg 2010; 39:889-896

10. Pettersson J, Kalbermatten D, McGrath A, et al. Biodegradable fibrin conduit promotes long-term regeneration after peripheral nerve injury in adult rats. J Plast Reconstr Aesthet Surg $2010 ; 63: 1893-1899$

11. Kalbermatten DF, Pettersson J, Kingham PJ, et al. New fibrin conduit for peripheral nerve repair. J Reconstr Microsurg 2009; 25:27-33

12. Lundborg $G$, Rosén $B$, Dahlin $L$, et al. Tubular versus conventional repair of median and ulnar nerves in the human forearm: early results from a prospective, randomized, clinical study. J Hand Surg Am 1997; 22:99-106

13. Zhang $\mathrm{P}$, Kou $\mathrm{Y}$, Yin X, et al. The experimental research of nerve fibers compensation amplification innervation of ular nerve and musculocutaneous nerve in rhesus monkeys. Artif Cells Blood Substit Immobil Biotechnol 2011; 39:39-43

14. Zhang $\mathrm{P}, \mathrm{He} X, \mathrm{Z}$ hoo $\mathrm{F}$, et al. Bridging small-gap peripheral nerve defects using biodegradable chitin conduits with cultured schwann and bone marrow stromal cells in rats. J Reconstr Microsurg 2005; 21:565-571 